\title{
Multilevel modelling of the risk of malaria among children aged under five years in Nigeria
}

\section{Victor M Oguoma ( $\nabla$ victor.oguoma@canberra.edu.au )}

Health Research Institute, University of Canberra, Canberra, Australia https://orcid.org/0000-00019505-7197

\section{Anayochukwu E Anyasodor}

School of Community Health, Charles Sturt University, Orange, Australia

\section{Adeniyi $O$ Adeleye}

School of Nursing, Midwifery and Social Sciences, Central Queensland University, Rockhampton,

Australia

\section{Obiora A Eneanya}

Washington University School of Medicine, Department of Medicine, Infectious Diseases Division, St.

Louis, Missouri, USA

\section{Evaristus C Mbanefo}

Bladder Immunology Group, Biomedical Research Institute, Rockville, Maryland, USA

\section{Research Article}

Keywords: Children under-five, Malaria infection, Parasitaemia, ITN, Nigeria, Sub-Saharan Africa

Posted Date: October 7th, 2020

DOI: https://doi.org/10.21203/rs.3.rs-32524/v2

License: (c) (i) This work is licensed under a Creative Commons Attribution 4.0 International License. Read Full License

Version of Record: A version of this preprint was published on September 18th, 2020. See the published version at https://doi.org/10.1093/trstmh/traa092. 


\section{Abstract}

\section{Background:}

Malaria is still a major cause of morbidity and mortality among children under five (U5) years. This study assessed individual, household, and community risk factors for malaria in Nigerian children U5 years of age.

\section{Methods:}

Data from the Nigerian Malaria Health Indicator Survey 2015 were pooled for analyses. This comprised of national survey of 329 clusters. Children aged 6-59 months who were tested for malaria using microscopy were retained. Multilevel logit model accounting for sampling design was used to assess individual, household and community factors associated with malaria parasitaemia.

\section{Results:}

A total of 5742 children were assessed for malaria parasitaemia with overall prevalence of $27 \%(95 \% \mathrm{Cl}$ 26-28\%). Plasmodium falciparum constituted $98 \%$ of the Plasmodium species. There was no significant difference in parasitaemia between older children, and those $\leq 12$ months. In adjusted analyses, rural living, Northwest region, household size of $>7$, dependence on river and rain water as primary water source were associated with higher odds of parasitaemia; while higher wealth index, all U5s who slept under bed net and dependence on packaged water were associated with lower odds of parasitaemia.

\section{Conclusion:}

Despite sustained investment in malaria control and prevention, a quarter of the overall study population of U5s suffer from malaria. Across the six geopolitical zones, the highest burden was in children living in poorest rural households.

\section{Introduction}

Malaria is a major public health problem in developing economies, especially in sub-Sahara Africa (SSA) where it is responsible for substantial morbidity and mortality (1) as well as other socioeconomic losses (2). The disease is caused by Plasmodium parasite, and transmitted through the bite of infected female Anopheles mosquitoes (3). It has been argued that the observed decline in All Cause Child Mortality is attributable to a commensurate trend in child malaria mortality in otherwise high risk areas (4-6). However, malaria still accounted for over 400,000 mortality in 2018, plus the fact that about 219 million cases of malaria occurred in 2017 compared to 217 million cases in 2016 (7). This report emphasises that no significant progress was made in reducing global malaria between the year 2015-2017. 
The African region of the World Health Organisation (WHO) contributes about $92 \%$ of malaria cases with South-East Asia (5\%) and the Eastern Mediterranean region (2\%) constituting the remaining fraction. Nigeria accounts for a quarter of the global malaria burden, far greater than Democratic Republic of the Congo, Mozambique, India and Uganda (7). Despite significant investments in the control of malaria, it remains a threat in Nigeria, especially among under-five (U5) children (8). In 2018, U5 children accounted for $67 \%$ and $61 \%$ of all malaria deaths worldwide and SSA, respectively. It is estimated that every two minutes, an U5 child dies of malaria, and SSA dominates in the death toll (9).

Understanding country-specific malaria risk, especially in the most vulnerable populations like U5 children will in no small measure form the prerequisite to establishing a long-term control intervention. In countries like Nigeria with less promising indices, the focus is still on case reduction, which requires investment in both human and material resources for surveillance and health system strengthening for any attempt at elimination (10). This study therefore assessed the relationship of demographic, socioeconomic and some environmental determinants of malaria transmission/prevention among U5 children at individual, household and community levels of disease intervention.

\section{Methods}

\section{Study area:}

The study was conducted in Nigeria, which is a country in West Africa. Nigeria shares border with Niger in the north, Chad in the northeast, Cameroon in the east and Benin Republic in the west. The south lies to the Gulf of Guinea in the Atlantic Ocean.

\section{Nigerian Malaria Indicator Survey data:}

The Nigerian Malaria Indicator Survey conducted in 2015 was implemented by the National Malaria Elimination Programme, the National Population Commission (NPC), the National Bureau of Statistics and the Malaria Partnership in Nigeria with technical assistance from ICF International. A nationally representative sample of over 8,000 households in 329 clusters were sampled between October and November 2015.

The sampling frame was based on the 2006 National Population and Housing Census of the Federal Republic of Nigeria, conducted by the NPC. Administratively, Nigeria is divided into states. Each state is subdivided into local government areas (LGAs), and each LGA is further divided into communities. In addition to these administrative units, during the 2006 census, each community was subdivided into convenient areas called census enumeration areas (EAs). The primary sampling unit, referred to as a cluster for the 2015 National Malaria Indicator Survey (NMIS), was defined based on EAs from the 2006 EA census frame. A two-stage sampling strategy was adopted. Nine clusters (EAs) were selected from each state, including the Federal Capital Territory (FCT) during the first stage in a manner that is 
representative of each state. The result was a total of 333 clusters throughout the country, 138 and 195 in urban and rural areas, respectively.

In the second stage of the selection process, 25 households were selected in each cluster by equal probability systematic sampling. All women aged 15-49 who were either permanent residents of the households in the 2015 NMIS sample or visitors present in the households on the night before the survey were eligible to be interviewed. In addition, all children age 6-59 months were eligible to be tested for malaria and anaemia. This sample size was selected to guarantee that key survey indicators could be produced for each of the country's six geopolitical zones, with approximately 1,338 women in each zone expected to complete interviews. In order to produce some of the survey indicators at the state level for each of the 36 states and the FCT, interviews were expected to be completed with approximately 217 women per state. A more detailed description of survey design and microscopy procedures can be found in the 2015 NMIS report (11).

\section{Malaria risk factors}

\section{Individual level explanatory variables:}

Gender, age and number of children who slept under insecticide treated bed net (ITN) a night before survey was considered potential individual level risk factors of malaria risk. We grouped age in months into five categories: $\leq 12$ months, $13-23$ months, $24-35$ months, 36-47 months and 48-59 months.

\section{Household level explanatory variables:}

This includes household size, comprising number of household members, mother's (caregiver's) level of education, number of U5 children in the household, wealth index level, household source of drinking water, the location of source of water and whether dwelling has been sprayed with insecticide(s) against mosquitoes. Household source of drinking water such as piped into dwelling, yard/plot, neighbour and public tap/standpipe were grouped together as piped; protected/unprotected well, protected/unprotected spring were grouped as well/spring; river/dam/lake/ponds/stream/canal/irrigation channel were grouped as river/stream; sachet water, bottled water, cart with small tank were grouped as packaged water; while borehole or tube well and rainwater stood alone forming six recategorised groups.

\section{Community level explanatory variables:}

The study considered place of residence (rural/urban) and region of residence as potential community level explanatory variables. The region of residence is based on the six geopolitical zones that exist in Nigeria-Northcentral, Northeast, Southwest, Southsouth and Southeast regions. 


\section{Ethical clearance}

Secondary data approved by ICF Macro International were used for this study. The ICF Macro International obtained ethical approval from ethical review boards of the Nigerian Ministry of Health before the survey commenced. Informed consent was received from the study participants before data collection. Also, participants were assured of the confidentiality and anonymity of the information provided during data collection.

\section{Statistical analysis}

Individual level data were retrieved from the nationally representative 2015 NMIS. The survey design estimation command (svy) in Stata 16.1 (StataCorp, College Station, TX) was used to conduct descriptive analysis, accounting for the sampling weight. Categorical variables are represented as counts and percentages. To assess the association between malaria in U5 children and individual, household, and community risk factors, we specified a three-level random intercept logistic regression model to account for contextual within-household and within-cluster correlations. Given the complex survey design of the data, sampling weight was included in the fixed effects portion of the model. The model is represented as below:

$$
Y_{i j k}=\beta_{0}+\beta_{1} X_{i j k}+\eta_{k}+\xi_{j k}
$$

Where $Y_{i j k}$ is the outcome (presence of malaria) for $i^{\text {th }}$ under 5 -year-old child at household $j$ in cluster $k . \beta_{0}$ is the mean log-odds of malaria across household and cluster. $X_{i j k}$ is a level 1 covariate for the $i^{\text {th }}$ child in household $j$ and cluster $k$, while $\beta_{1}$ represents the slope associated with $X_{i j k}$ which represents the relationship between the level 1 covariates and the log odds of malaria. $\eta_{k}$ is the random effect for cluster, while $\xi_{i j}$ is the household random effect. The random effects are independent and identical to each other and are assumed to be normally distributed with mean of zero and variances $\sigma_{\eta}$ and $\sigma_{\xi}$.

A univariate multi-level logistic regression was conducted, using each individual, household, and community risk factors as predictors and malaria risk as outcome. Individual predictors with any level less than $p=0.10$ were considered for inclusion in the multivariable multi-level logistic regression. The multivariable analysis was conducted in a sequential process. Firstly, we fitted a null model (Model 0 ) -an unconditional model-to decompose the total variance of malaria prevalence between the cluster and level 1 covariates. Model 1 consist of individual level factors, Model 2 included household level factors, while model 3 included community level factors to constitute a fully adjusted model.

The influence of contextual household and cluster effects on the prevalence of malaria were explored, and expressed as the area variance, intracluster correlation and the median odds ratio $(12,13)$. Estimates of the univariate association between outcomes and individual, household or community level covariates are reported as odds ratio (OR) and the $95 \%$ confidence interval, while the multivariable models (Models 
1, 2 and 3) are reported as adjusted odds ratio (AOR) and $95 \% \mathrm{Cl}$. The goodness of fit of the sequential multivariable models were assessed using the Akaike Information Criterion (AIC). Statistical significance was set at an alpha level 0.05 .

\section{Results}

\section{Characteristics of study population and prevalence of malaria}

Table 1. There was a total number of 5742 (weighted $=5814$ ) children in 3685 households, and 326 clusters with valid record for malaria parasitaemia assessed through microscopy. The mean age of U5 children assessed for malaria parasitaemia was 36 months (range: 0 - 59 months), of which 50\% were females. About $43 \%$ of the children were reported to have slept under mosquito bet net last night before the interview, while $42.6 \%$ of the caregivers have completed no education. Majority of the household source of drinking water were from borehole/ tube well (34.4\%) and well/spring water (30.4\%). Overall, only $1.3 \%$ had dwelling place sprayed against mosquito in the last 12 months, and $65.9 \%$ of the children lived in rural areas. The overall malaria prevalence was $27.3 \%$ (95\% Cl: $24.8-30.0 \%)$. The prevalence of Plasmodium species - falciparum, malariae and ovale were $98.2 \%, 5 \%$ and $1.1 \%$, respectively.

Figure 1 shows the prevalence of U5 malaria based on source of drinking water across regions. Well/ spring and borehole/tube well are the predominant sources of drinking water in the northern regions amongst U5 children with malaria. About $23.8 \%$ prevalence of U5 malaria in Northwest was as a result of use of well/spring sources of drinking water.

Table 1. Study characteristics 


\begin{tabular}{|c|c|c|}
\hline & \multicolumn{2}{|c|}{ Total population } \\
\hline & Unweighted & Weighted \\
\hline & $\mathrm{N}=5742$ & $\mathrm{~N}=5814$ \\
\hline \multicolumn{3}{|l|}{ Child's age in months } \\
\hline$\leq 12$ & $744 / 5388(13.8)$ & $722 / 5470(13.2)$ \\
\hline $13-23$ & $597 / 5388(11.1)$ & $618 / 5470(11.3)$ \\
\hline $24-35$ & $853 / 5388(15.8)$ & $805 / 5470(14.7)$ \\
\hline $36-47$ & $1469 / 5388(27.3)$ & $1564 / 5470(28.6)$ \\
\hline $48-59$ & $1725 / 5388(32.0)$ & $1760 / 5470(32.2)$ \\
\hline \multicolumn{3}{|l|}{ Sex of child } \\
\hline Male & $2833 / 5742(49.3)$ & $2894 / 5814(49.8)$ \\
\hline Female & $2909 / 5742(50.7)$ & $2921 / 5814(50.2)$ \\
\hline \multicolumn{3}{|c|}{$\begin{array}{l}\text { Children under } 5 \text { slept under } \\
\text { mosquito bed net last night }\end{array}$} \\
\hline No & $1145 / 4188(27.3)$ & $1047 / 4184(25.0)$ \\
\hline All children & $1769 / 4188(42.2)$ & $1816 / 4184(43.4)$ \\
\hline Some children & $425 / 4188(10.1)$ & $471 / 4184(11.3)$ \\
\hline No net in household & $849 / 4188(20.3)$ & $850 / 4184(20.3)$ \\
\hline \multicolumn{3}{|c|}{ Highest educational level } \\
\hline No education & $2312 / 5742(40.3)$ & $2479 / 5814(42.6)$ \\
\hline Primary & $900 / 5742(15.7)$ & $880 / 5814(1514)$ \\
\hline Secondary & $2111 / 5742(36.8)$ & $2026 / 5814(34.8)$ \\
\hline Higher & $419 / 5742(7.3)$ & $429 / 5814(7.4)$ \\
\hline \multicolumn{3}{|c|}{ Number of household members } \\
\hline$<5$ & $1243 / 5742(21.6)$ & $1313 / 5814(22.6)$ \\
\hline $5-7$ & $2422 / 5742(42.2)$ & $2406 / 5814(41.4)$ \\
\hline$>7$ & $2077 / 5742(36.2)$ & $2095 / 5814(36.0)$ \\
\hline \multicolumn{3}{|c|}{$\begin{array}{l}\text { Number of children } 5 \text { and under } \\
\text { in household }\end{array}$} \\
\hline 0 & $1366 / 5742(23.8)$ & $1385 / 5814(23.8)$ \\
\hline $1-2$ & $3267 / 5742(56.9)$ & $3346 / 5814(57.5)$ \\
\hline$>2$ & $1109 / 5742(19.3)$ & $1083 / 5814(18.6)$ \\
\hline \multicolumn{3}{|l|}{ Wealth index } \\
\hline Poorest & $1006 / 5742(17.5)$ & $1074 / 5814(18.5)$ \\
\hline Poorer & $1266 / 5742(22.0)$ & $1352 / 5814(23.3)$ \\
\hline Middle & $1261 / 5742(22.0)$ & $1229 / 5814(21.1)$ \\
\hline Richer & $1148 / 5742(20.0)$ & $1128 / 5814(19.4)$ \\
\hline Richest & $1061 / 5742(18.5)$ & $1032 / 5814(17.7)$ \\
\hline \multicolumn{3}{|c|}{ Source of drinking water } \\
\hline Piped & $609 / 5742(10.6)$ & $665 / 5814(11.4)$ \\
\hline Borehole tube well & $2033 / 5742(35.4)$ & $1998 / 5814(34.4)$ \\
\hline Well/spring & $1689 / 5742(29.4)$ & $1767 / 5814(30.4)$ \\
\hline River/stream & $649 / 5742(11.3)$ & $632 / 5814(10.9)$ \\
\hline Rainwater & $204 / 5742(3.6)$ & $191 / 5814(3.3)$ \\
\hline Packaged water & $558 / 5742(9.7)$ & $562 / 5814(9.7)$ \\
\hline \multicolumn{3}{|c|}{ Location of source for water } \\
\hline In own dwelling & $934 / 5,389(17.3 \%)$ & $905 / 5454(16.6)$ \\
\hline In own yard/plot & $664 / 5,389(12.3 \%)$ & $692 / 5454(12.7)$ \\
\hline Elsewhere & $3,791 / 5,389(70.3 \%)$ & $3858 / 5454(70.7)$ \\
\hline $\begin{array}{l}\text { Dwelling sprayed } \\
\text { against mosquitoes }\end{array}$ & & \\
\hline
\end{tabular}




\begin{tabular}{|l|l|l|} 
No & $5605 / 5742(97.6)$ & $5700 / 5814(98.0)$ \\
\hline Yes & $93 / 5742(1.6)$ & $78 / 5814(1.3)$ \\
\hline Don't know & $44 / 5742(0.8)$ & $37 / 5814(0.6)$ \\
\hline Place of residence & & \\
\hline Urban & $1978 / 5742(34.4)$ & $1984 / 5814(34.1)$ \\
\hline Rural & $3764 / 5742(65.6)$ & $3831 / 5814(65.9)$ \\
\hline Region & & \\
\hline Northcentral & $1160 / 5742(20.2)$ & $1115 / 5814(19.2)$ \\
\hline Northeast & $1118 / 5742(19.5)$ & $798 / 5814(13.7)$ \\
\hline Northwest & $1423 / 5742(24.8)$ & $1843 / 5814(31.7)$ \\
\hline Southeast & $568 / 5742(9.9)$ & $511 / 5814(8.8)$ \\
\hline Southsouth & $741 / 5742(12.9)$ & $654 / 5814(11.3)$ \\
\hline Southwest & $732 / 5742(12.7)$ & $893 / 5814(15.4)$ \\
\hline Malaria from blood smear test & & \\
\hline Negative & $4,179 / 5,742(72.8 \%)$ & $4225 / 5814(72.7)$ \\
\hline Positive & $1,563 / 5,742(27.2 \%)$ & $1590 / 5814(27.3)$ \\
\hline Presence of Plasmodium falciparum & & \\
\hline No & $24 / 1,563(1.5)$ & $29 / 1590(1.8)$ \\
\hline Yes & $1,539 / 1,563(98.5)$ & $1560 / 1590(98.2)$ \\
\hline Presence of Plasmodium malariae & & \\
\hline No & $1,484 / 1,563(94.9)$ & $1510 / 15 / 90(95.0)$ \\
\hline Yes & $79 / 1,563(5.1)$ & $80 / 1590(5.0)$ \\
\hline Presence of Plasmodium ovale & & $1572 / 1590(98.9)$ \\
\hline No & $1,543 / 1,563(98.7)$ & $18 / 1590(1.1)$ \\
\hline Yes & $20 / 1,563(1.3)$ & \\
\hline & & \\
\hline & & \\
\hline
\end{tabular}

\section{Association of malaria parasitaemia with individual, household and community level factors}

Table 2. The prevalence of malaria parasitaemia was $85.8 \%$ in children living in rural areas compared to $14.2 \%$ in the urban areas. Under 5 children in the Northwestern region had the highest prevalence of malaria parasitaemia (43.1\%), followed by Northcentral (22.6\%), Northeast (13.3\%), Southwest (8.6\%), Southsouth (8.0\%) and Southeast (4.4\%). The highest prevalence of malaria was recorded in children aged 48-59 months (33.4\%) and lowest in 13-23 months old.

Table 2. Univariate association of malaria risk and study characteristics 


\begin{tabular}{|c|c|c|c|}
\hline & $\begin{array}{l}\text { Malaria positive } \\
(\mathrm{N}=1590) \\
\text { Weighted } \mathrm{n}(\%)\end{array}$ & OR $(95 \% \mathrm{CI}) \$$ & p-value \\
\hline \multicolumn{4}{|l|}{ Sex of child } \\
\hline Male & $706(44.40)$ & $1.00(1.00,1.00)$ & \\
\hline Female & $884(55.60)$ & $1.18(0.86,1.62)$ & 0.30 \\
\hline \multicolumn{4}{|l|}{ Child's age in months } \\
\hline$\leq 12$ & $194(13.43)$ & $1.00(1.00,1.00)$ & \\
\hline $13-23$ & $142(9.83)$ & $0.63(0.33,1.21)$ & 0.16 \\
\hline $24-35$ & $180(12.46)$ & $0.62(0.35,1.08)$ & 0.09 \\
\hline $36-47$ & $447(30.84)$ & $0.94(0.58,1.54)$ & 0.82 \\
\hline $48-59$ & $484(33.43)$ & $1.02(0.62,1.67)$ & 0.95 \\
\hline \multicolumn{4}{|c|}{$\begin{array}{l}\text { Children under } 5 \text { slept under } \\
\text { mosquito bed net last night }\end{array}$} \\
\hline no & $243(20.46)$ & $1.00(1.00,1.00)$ & \\
\hline all children & $531(44.63)$ & $1.04(0.64,1.69)$ & 0.88 \\
\hline some children & $194(16.32)$ & $2.79(1.39,5.60)$ & 0.004 \\
\hline no net in household & $221(18.60)$ & $1.08(0.64,1.83)$ & 0.78 \\
\hline \multicolumn{4}{|c|}{ Number of household members } \\
\hline$<5$ & $282(17.72)$ & $1.00(1.00,1.00)$ & \\
\hline $5-7$ & $656(41.24)$ & $1.30(0.98,1.73)$ & 0.07 \\
\hline$>7$ & $652(41.03)$ & $1.60(1.18,2.16)$ & 0.003 \\
\hline \multicolumn{4}{|c|}{ Highest educational level } \\
\hline No education & $983(61.82)$ & $1.00(1.00,1.00)$ & \\
\hline Primary & $220(13.85)$ & $0.42(0.26,0.68)$ & $<0.001$ \\
\hline Secondary & $313(19.70)$ & $0.21(0.14,0.32)$ & $<0.001$ \\
\hline Higher & $74(4.64)$ & $0.14(0.07,0.29)$ & $<0.001$ \\
\hline \multicolumn{4}{|c|}{ Number of children 5 and under in household } \\
\hline 0 & $338(21.24)$ & $1.00(1.00,1.00)$ & \\
\hline $1-2$ & $892(56.08)$ & $0.92(0.60,1.40)$ & 0.69 \\
\hline$>2$ & $361(22.68)$ & $1.08(0.65,1.78)$ & 0.78 \\
\hline \multicolumn{4}{|l|}{ Wealth index } \\
\hline Poorest & $467(29.40)$ & $1.00(1.00,1.00)$ & \\
\hline Poorer & $519(32.64)$ & $0.82(0.52,1.28)$ & 0.38 \\
\hline Middle & $373(23.45)$ & $0.57(0.34,0.95)$ & 0.03 \\
\hline Richer & $154(9.70)$ & $0.16(0.09,0.28)$ & $<0.001$ \\
\hline Richest & $76(4.80)$ & $0.05(0.03,0.11)$ & $<0.001$ \\
\hline \multicolumn{4}{|c|}{ Source of drinking water } \\
\hline Piped & $112(7.02)$ & $1.00(1.00,1.00)$ & \\
\hline Borehole & $485(30.51)$ & $1.42(0.87,2.31)$ & 0.16 \\
\hline Well/spring & $684(43.05)$ & $2.92(1.76,4.85)$ & $<0.001$ \\
\hline River/stream & $233(14.65)$ & $2.90(1.64,5.13)$ & $<0.001$ \\
\hline Rainwater & $47(2.95)$ & $2.25(1.06,4.75)$ & 0.03 \\
\hline Packaged water & $29(1.83)$ & $0.34(0.18,0.63)$ & $<0.001$ \\
\hline \multicolumn{4}{|c|}{ Location of source for water } \\
\hline In own dwelling & $225(14.69)$ & $1.00(1.00,1.00)$ & \\
\hline In own yard/plot & $186(12.12)$ & $1.12(0.71,1.77)$ & 0.63 \\
\hline Elsewhere & $1120(73.19)$ & $1.03(0.69,1.53)$ & 0.90 \\
\hline \multicolumn{4}{|c|}{$\begin{array}{l}\text { Dwelling sprayed against mosquitoes } \\
\text { in last } 12 \text { months }\end{array}$} \\
\hline No & $1566(98.49)$ & $1.00(1.00,1.00)$ & \\
\hline
\end{tabular}




\begin{tabular}{|l|l|l|l|} 
Yes & $15(0.95)$ & $1.06(0.38,2.96)$ & 0.91 \\
\hline Don't know & $9(0.56)$ & $1.63(0.47,5.64)$ & 0.44 \\
\hline Place of residence & & & \\
\hline Urban & $226(14.22)$ & $1.00(1.00,1.00)$ & \\
\hline Rural & $1364(85.78)$ & $8.66(5.23,14.35)$ & $<0.001$ \\
\hline Region & & & \\
\hline Northcentral & $359(22.60)$ & $1.00(1.00,1.00)$ & \\
\hline Northeast & $211(13.27)$ & $1.13(0.53,2.43)$ & 0.76 \\
\hline Northwest & $685(43.10)$ & $3.34(1.62,6.86)$ & $\mathbf{0 . 0 0 1}$ \\
\hline Southeast & $70(4.42)$ & $0.26(0.11,0.60)$ & $\mathbf{0 . 0 0 2}$ \\
\hline Southsouth & $127(7.97)$ & $0.53(0.24,1.17)$ & 0.12 \\
\hline Southwest & $137(8.64)$ & $0.49(0.22,1.12)$ & 0.09 \\
\hline
\end{tabular}

\$ Estimated from univariate multilevel logistic regression

Table 3 shows results of the multivariable multilevel logistic regression analyses. At the individual level factors, all children in a household who slept under ITN in previous night had a lower odd of malaria [AOR=0.60 (95\% Cl: 0.38-0.94; $\mathrm{p}=0.03$ )] compared to those who did not sleep under ITN (adjusted Model 3 ). At the household-level, children in households with members $>7$ had $47 \%$ greater odd of malaria, compared to those with $<5$ members after accounting for other individual, household and community level factors. Children whose caregivers have primary or secondary education had lower risk of malaria compared to those born to a non-educated caregiver. The higher the wealth index of the household, the lower the odds of U5 malaria parasitaemia. Also, households whose source of drinking water is from well/spring water, rainwater, and river had 2.45, 2.79, and 3.03-fold increase in the odds of malaria parasitaemia compared to those who drink from pipe borne water, respectively. Conversely, children from household that use packaged water as source of drinking had significantly lower odds of malaria. At the community level, children who live in the rural areas had 2.68-fold increase in the odds of malaria compared to those who live in urban areas. However, children from the Northwest region had significantly 2.35-fold increase in odds of malaria compared to those from Northcentral region.

Figure 2 shows the predicted probabilities of malaria risk for children living in households with varying wealth index and in rural/urban areas across the six geopolitical regions. Although, children in Northwest region had the highest probability of malaria parasitaemia infection for each household wealth index and place of residence, the trend seem to be the same for children at all regions.

Table 3. Multivariable multilevel logistic regression of malaria risk across study characteristics 


\begin{tabular}{|c|c|c|c|c|c|c|}
\hline Characteristics & Model 1 & & Model 2 & & Model 3 & \\
\hline & AOR (95\%CI) & $P$ & AOR (95\%CI) & $P$ & AOR (95\%CI) & $P$ \\
\hline \multicolumn{7}{|c|}{ Individual level factors } \\
\hline \multicolumn{7}{|c|}{ Sex of child } \\
\hline Male & $\begin{array}{l}1.00(1.00, \\
1.00)\end{array}$ & & $\begin{array}{l}1.00(1.00, \\
1.00)\end{array}$ & & $\begin{array}{l}1.00(1.00, \\
1.00)\end{array}$ & \\
\hline Female & $\begin{array}{l}0.99(0.67, \\
1.47)\end{array}$ & 0.98 & $\begin{array}{l}1.03(0.73, \\
1.46)\end{array}$ & 0.85 & $\begin{array}{l}1.01(0.72, \\
1.43)\end{array}$ & 0.95 \\
\hline \multicolumn{7}{|l|}{ Child's age in months } \\
\hline$\leq 12$ & $\begin{array}{l}1.00(1.00, \\
1.00)\end{array}$ & & $\begin{array}{l}1.00(1.00, \\
1.00)\end{array}$ & & $\begin{array}{l}1.00(1.00, \\
1.00)\end{array}$ & \\
\hline $13-23$ & $\begin{array}{l}0.66(0.33, \\
1.35)\end{array}$ & 0.26 & $\begin{array}{l}0.61(0.31, \\
1.19)\end{array}$ & 0.15 & $\begin{array}{l}0.63(0.32, \\
1.24)\end{array}$ & 0.18 \\
\hline $24-35$ & $\begin{array}{l}0.67(0.37, \\
1.22)\end{array}$ & 0.19 & $\begin{array}{l}0.62(0.35, \\
1.11)\end{array}$ & 0.11 & $\begin{array}{l}0.65(0.37, \\
1.17)\end{array}$ & 0.15 \\
\hline $36-47$ & $\begin{array}{l}0.98(0.57, \\
1.68)\end{array}$ & 0.94 & $\begin{array}{l}0.77(0.46, \\
1.29)\end{array}$ & 0.32 & $\begin{array}{l}0.77(0.46, \\
1.28)\end{array}$ & 0.31 \\
\hline $48-59$ & $\begin{array}{l}0.87(0.50, \\
1.53)\end{array}$ & 0.63 & $\begin{array}{l}0.70(0.42, \\
1.18)\end{array}$ & 0.18 & $\begin{array}{l}0.70(0.41, \\
1.18)\end{array}$ & 0.18 \\
\hline \multicolumn{7}{|c|}{$\begin{array}{l}\text { Children under } 5 \text { slept under } \\
\text { mosquito bed net last night }\end{array}$} \\
\hline No & $\begin{array}{l}1.00(1.00, \\
1.00)\end{array}$ & & $\begin{array}{l}1.00(1.00, \\
1.00)\end{array}$ & & $\begin{array}{l}1.00(1.00, \\
1.00)\end{array}$ & \\
\hline All children & $\begin{array}{l}0.96(0.58, \\
1.60)\end{array}$ & 0.89 & $\begin{array}{l}0.61(0.39, \\
0.96)\end{array}$ & 0.03 & $\begin{array}{l}0.60(0.38, \\
0.94)\end{array}$ & 0.03 \\
\hline Some children & $\begin{array}{l}2.83(1.35 \\
5.94)\end{array}$ & 0.01 & $\begin{array}{l}1.52(0.76, \\
3.07)\end{array}$ & 0.24 & $\begin{array}{l}1.41(0.70 \\
2.85)\end{array}$ & 0.33 \\
\hline No net in household & $\begin{array}{l}1.05(0.61, \\
1.80)\end{array}$ & 0.87 & $\begin{array}{l}1.05(0.67, \\
1.65)\end{array}$ & 0.83 & $\begin{array}{l}1.14(0.73, \\
1.79)\end{array}$ & 0.57 \\
\hline \multicolumn{7}{|c|}{ Household level factors } \\
\hline \multicolumn{7}{|c|}{$\begin{array}{l}\text { Number of household } \\
\text { members }\end{array}$} \\
\hline$<5$ & & & $\begin{array}{l}1.00(1.00, \\
1.00)\end{array}$ & & $\begin{array}{l}1.00(1.00, \\
1.00)\end{array}$ & \\
\hline $5-7$ & & & $\begin{array}{l}1.14(0.80, \\
1.62)\end{array}$ & 0.47 & $\begin{array}{l}1.14(0.80, \\
1.63)\end{array}$ & 0.45 \\
\hline$>7$ & & & $\begin{array}{l}1.45(1.01, \\
2.10)\end{array}$ & 0.05 & $\begin{array}{l}1.47(1.02, \\
2.13)\end{array}$ & 0.04 \\
\hline \multicolumn{7}{|c|}{ Highest educational level } \\
\hline No education & & & $\begin{array}{l}1.00(1.00, \\
1.00)\end{array}$ & & $\begin{array}{l}1.00(1.00, \\
1.00)\end{array}$ & \\
\hline Primary & & & $\begin{array}{l}0.56(0.34, \\
0.93)\end{array}$ & 0.03 & $\begin{array}{l}0.59(0.35, \\
0.97)\end{array}$ & 0.04 \\
\hline Secondary & & & $\begin{array}{l}0.67(0.41, \\
1.10)\end{array}$ & 0.12 & $\begin{array}{l}0.70(0.42, \\
1.16)\end{array}$ & 0.17 \\
\hline Higher & & & $\begin{array}{l}1.10(0.44, \\
2.73)\end{array}$ & 0.83 & $\begin{array}{l}1.06(0.43, \\
2.60)\end{array}$ & 0.90 \\
\hline \multicolumn{7}{|l|}{ Wealth index } \\
\hline $\begin{array}{l}\text { Poorest } \\
\end{array}$ & & & $\begin{array}{l}1.00(1.00, \\
1.00)\end{array}$ & & $\begin{array}{l}1.00(1.00, \\
1.00)\end{array}$ & \\
\hline Poorer & & & $\begin{array}{l}0.89(0.52, \\
1.52)\end{array}$ & 0.67 & $\begin{array}{l}1.03(0.60, \\
1.78)\end{array}$ & 0.91 \\
\hline
\end{tabular}




\begin{tabular}{|c|c|c|c|c|}
\hline Middle & $\begin{array}{l}0.71(0.39, \\
1.28)\end{array}$ & 0.25 & $\begin{array}{l}0.89(0.49, \\
1.64)\end{array}$ & 0.71 \\
\hline Richer & $\begin{array}{l}0.22(0.11, \\
0.47)\end{array}$ & $<0.001$ & $\begin{array}{l}0.34(0.16, \\
0.74)\end{array}$ & 0.01 \\
\hline Richest & $\begin{array}{l}0.08(0.03, \\
0.20)\end{array}$ & $<0.001$ & $\begin{array}{l}0.15(0.06, \\
0.38)\end{array}$ & $<0.001$ \\
\hline \multicolumn{5}{|c|}{ Source of drinking water } \\
\hline Piped & $\begin{array}{l}1.00(1.00, \\
1.00)\end{array}$ & & $\begin{array}{l}1.00(1.00, \\
1.00)\end{array}$ & \\
\hline Borehole & $\begin{array}{l}1.07(0.63, \\
1.80)\end{array}$ & 0.80 & $\begin{array}{l}1.18(0.68, \\
2.04)\end{array}$ & 0.56 \\
\hline Well/spring & $\begin{array}{l}2.45(1.40 \\
4.29)\end{array}$ & 0.002 & $\begin{array}{l}2.45(1.35, \\
4.43)\end{array}$ & 0.003 \\
\hline River/stream & $\begin{array}{l}2.82(1.48, \\
5.35)\end{array}$ & 0.002 & $\begin{array}{l}3.03(1.56, \\
5.89)\end{array}$ & 0.001 \\
\hline Rainwater & $\begin{array}{l}2.57(1.07 \\
6.16)\end{array}$ & 0.035 & $\begin{array}{l}2.79(1.13, \\
6.93)\end{array}$ & 0.03 \\
\hline Packaged water & $\begin{array}{l}0.39(0.19, \\
0.77)\end{array}$ & 0.007 & $\begin{array}{l}0.43(0.21, \\
0.86)\end{array}$ & 0.02 \\
\hline \multicolumn{5}{|c|}{ Community level factors } \\
\hline \multicolumn{5}{|c|}{ Place of residence } \\
\hline Urban & & & $\begin{array}{l}1.00(1.00, \\
1.00)\end{array}$ & \\
\hline Rural & & & $\begin{array}{l}2.68(1.51, \\
4.77)\end{array}$ & $<0.001$ \\
\hline \multicolumn{5}{|l|}{ Region } \\
\hline Northcentral & & & $\begin{array}{l}1.00(1.00, \\
1.00)\end{array}$ & \\
\hline Northeast & & & $\begin{array}{l}0.92(0.47, \\
1.78)\end{array}$ & 0.8 \\
\hline Northwest & & & $\begin{array}{l}2.35(1.18, \\
4.69)\end{array}$ & 0.02 \\
\hline Southeast & & & $\begin{array}{l}1.43(0.60, \\
3.41)\end{array}$ & 0.43 \\
\hline Southsouth & & & $\begin{array}{l}1.25(0.56, \\
2.78)\end{array}$ & 0.59 \\
\hline Southwest & & & $\begin{array}{l}1.98(0.90, \\
4.34)\end{array}$ & 0.09 \\
\hline
\end{tabular}

\section{Measures of household and cluster-level variation in malaria risk}

Estimates of the cluster and household random effects (Table 4) show significant variation in the prevalence of U5 malaria at the cluster level (3.87 in the null model to 1.74) after accounting for individual, household and community-level fixed effect factors. This shows that $54.8 \%$ of the contextual cluster level variance for malaria parasitaemia can be explained by individual, household and community level fixed effect predictors. A graph of the cluster and averaged State random effects show the variations of malaria risk by cluster (A) and State (B) from the null model (Figure 3). 
Table 4. Measures of cluster and household level variations

\begin{tabular}{|c|c|c|c|c|c|c|c|c|}
\hline & Null model & & Model 1 & & Model 2 & & Model 3 & \\
\hline $\begin{array}{l}\text { Random effects } \\
\text { measures of variation }\end{array}$ & $\begin{array}{l}\text { Estimate } \\
(95 \% \mathrm{CI})\end{array}$ & $P$ & $\begin{array}{l}\text { Estimate } \\
(95 \% \mathrm{CI})\end{array}$ & $P$ & $\begin{array}{l}\text { Estimate } \\
(95 \% \mathrm{CI})\end{array}$ & $P$ & $\begin{array}{l}\text { Estimate } \\
(95 \% \mathrm{CI})\end{array}$ & $P$ \\
\hline \multicolumn{9}{|l|}{ Area variance } \\
\hline Cluster level & $\begin{array}{l}3.87(2.73, \\
5.00)\end{array}$ & $<0.001$ & $\begin{array}{l}3.57(2.25, \\
4.89)\end{array}$ & $<0.001$ & $\begin{array}{l}1.79 \\
(1.12, \\
2.46)\end{array}$ & $<0.001$ & $\begin{array}{l}1.76 \\
(1.08 \\
2.43)\end{array}$ & $<0.001$ \\
\hline Household-level & $\begin{array}{l}2.08(1.18, \\
2.98)\end{array}$ & $<0.001$ & $\begin{array}{l}2.26(1.10, \\
3.41)\end{array}$ & $<0.001$ & $\begin{array}{l}2.17 \\
(1.08, \\
3.25)\end{array}$ & $<0.001$ & $\begin{array}{l}2.18 \\
(1.08, \\
3.27)\end{array}$ & $<0.001$ \\
\hline \multicolumn{9}{|l|}{$\begin{array}{l}\text { Intracluster } \\
\text { correlation }\end{array}$} \\
\hline Cluster level & $\begin{array}{l}0.42(0.36, \\
0.48)\end{array}$ & - & $\begin{array}{l}0.39(0.32, \\
0.47)\end{array}$ & - & $\begin{array}{l}0.25 \\
(0.19, \\
0.31)\end{array}$ & - & $\begin{array}{l}0.24 \\
(0.19, \\
0.31)\end{array}$ & - \\
\hline Household-level & $\begin{array}{l}0.64(0.57, \\
0.71)\end{array}$ & - & $\begin{array}{l}0.64(0.55, \\
0.72)\end{array}$ & - & $\begin{array}{l}0.55 \\
(0.45, \\
0.64)\end{array}$ & - & $\begin{array}{l}0.54 \\
(0.45, \\
0.64)\end{array}$ & - \\
\hline \multicolumn{9}{|l|}{ Median odds ratio } \\
\hline Cluster level & 10.88 & - & 9.76 & - & 4.47 & - & 4.40 & - \\
\hline Household-level & 5.19 & - & 5.64 & - & 5.41 & - & 5.43 & - \\
\hline \multicolumn{9}{|l|}{ Model fit } \\
\hline $\mathrm{AIC}$ & 5615 & - & 3900 & - & 3792 & - & 3786 & - \\
\hline
\end{tabular}

AIC: Akaike Information Criterion

\section{Discussion}

This representative study of Nigerian children U5 years old shows a high prevalence of malaria in this vulnerable group. Our result shows $27 \%$ prevalence of U5 malaria diagnosed by microscopy was found in Nigeria. This reveals a fraction of the huge national burden of malaria in Nigeria. Over $93 \%$ of malaria cases and related deaths were recorded in Africa, with Nigeria ranking up there as the most endemic country with $25 \%$ and $24 \%$ of global malaria cases and deaths, respectively (7).

While no association was recorded for age and sex, we found that the highest prevalence of malaria among U5 children in our sample population was recorded in children aged 4-5 years [48-59 months] (33.4\%). The absence of definite stratification within this age group suggests that all children under 5 years of age are at high risk. In fact, a seasonal malaria chemoprevention program has been piloted, and shown to significantly reduce malaria in under 5 children participating in the mass drug administration program in different areas of diverse malaria transmission intensity (14-17). Some economic benefit has further been attributed to seasonal malaria chemoprevention of all under 5 children $(16,18)$, with recommendation for this approach to be integrated into the health system in endemic areas.

The study did not observe any sex difference in U5 malaria prevalence in Nigeria. Our finding is in agreement with other studies in U5 children in Ethiopia (19) and Burkina Faso (20). In addition to demographics, another individual level factor assessed was the availability and use of ITNs by U5 
children, the night before the survey. Although the prevalence of malaria infection decreased when at least some children slept under the ITNs, there was no definite association between use of ITNs the night before and U5 malaria in the study population. While other studies had reported up to 10-fold decrease in under 5 malaria due to use of ITNs (19), our data suggest that the use of ITNs alone may not be sufficient for effective malaria control and elimination as opined by others (21).

At the household level, we found strong association between several assessed demographic and socioeconomic determinants. High number of household members but not the number of U5 children significantly increased malaria risk, especially when this number exceeds seven members. As previous studies have found that Anopheles mosquitoes are attracted to higher concentration of carbon dioxide as well as human odour (22), it is therefore plausible that larger households in our data are more likely to have an increased risk of mosquito bites.

Furthermore, our data suggest that children with educated mothers had less odds of malaria. As mother's education is often used as a proxy for household wealth (23), previous studies have found a reduced risk of malaria in wealthier households (24). There is the consistent trend in association between the household wealth index, an indicator related to other household determinants that impact on malaria incidence, with the poor suffering the brunt of malaria cases and perhaps mortality. Maternal education profoundly affects the household perception of malaria preventive measures, including acceptability and practice of malaria control interventions (25). Putative causal relationship has been reported for the impact of mother's level of education to U5 malaria (26). Wealth index is closely related to educational level and has been shown in this study to reduce malaria risks, consistently showing a negative association between household wealth and risk of under 5 malaria. Because wealth impacts other indices like education, housing, household nutrition, area of residence and health seeking behaviour, it is arguably a major determinant of U5 malaria $(25,27)$. Although malaria can be rightly described as a disease of poverty (28), no association was found in some studies between chronic malnutrition and U5 malaria (29), suggesting a more complex relationship.

Also, household domestic water sources that promote availability of stagnant water will directly impact the breeding capacity of malaria vectors, indirectly worsening malaria transmission among U5 children. Potable water resources and indoor residual spraying are key malaria intervention efforts targeted at the disease vectors that has recorded significant success in malaria transmission in several diverse settings $(25,30,31)$. Indeed, termination of indoor residual spraying intervention was reported in a study to result in rebound of prevalence to epidemic proportion (32).

At the community level, residence in urban or rural areas can impact malaria infection in U5 children mainly related to associated factors like population density, proximity to favourable vector breeding sites, agricultural projects, and closeness to health facilities. Like other studies, we also found that residence in rural areas and suburbs can worsen malaria transmission among U5 children $(33,34)$. The low prevalence in urban areas may be related to population density and its impact on the efficacy of malaria control interventions. Stebbins et al (35) reported variation in the efficacy of ITNs in urban and rural areas, 
concluding that ITNs use in urban areas offer benefits beyond individual protection. This further implicates population density factors, which were not observed in rural settings with more sparse population. Children U5 in the Northwest region had the highest odds of U5 malaria. They account for the highest proportion of reliance on open well and spring water for their source of drinking water. Such water bodies are known for providing suitable breeding sites for the malaria vector.

However, urban and rural areas in SSA have starkly varying types of housing. Tusting et al implemented a geospatial model to estimate the prevalence of improved housing in Africa (36). Their study defined 'improved housing' as houses built with finished materials such as brick and cement, whereas 'unimproved houses' were houses built with natural materials such as mud, thatch and less likely to have door and window screens as well as suitable furniture for mounting ITNs. Although there has been significant improvement in housing type in the past decade, there remains vast areas with unimproved housing, mostly in rural settlements. Previous studies have also found that malaria risk is higher in rural areas (37).

The multilevel results show significant variation in the risk of U5 malaria across sampled clusters and to some lesser extent across households. About $54.8 \%$ of the contextual cluster level variance for malaria parasitaemia can be explained by individual, household and community level fixed effect predictors. Averaging of the cluster-level variations by State shows that the contextual variations in U5 malaria risk are lower in most States in the southern region and higher in States in the Northern region. The sharp decrease in the cluster level median odds ratio after controlling for individual, household and communitylevel factors, again signify the important role these factors play in malaria transmission/intervention. While a spatially explicit analysis was not performed in this work, there is likely a spatial pattern in our prevalence estimates, given that Nigeria has distinct climate, socio-economic and environmental characteristics between its region. We therefore believe that these are significant contributing factors to regional variations in U5 malaria transmission. It will be interesting to thoroughly investigate these regional variations in U5 malaria transmission within a spatial framework.

\section{Strengths And Limitations}

The large and nationally representative study population is an important strength of the study. Parasitaemia was diagnosed through microscopy, which is the gold standard. Also, the study was able to identify individual, household and community level predictors of U5 malaria in Nigeria, thus provides information that will favour design of suitable intervention. Given that the data resulted from crosssectional designs, causal relationship between explanatory variables and U5 parasitaemia cannot be assumed.

\section{Conclusions}

Malaria remains a substantial public health burden in Nigeria, especially in U5 children despite sustained investment in malaria control and prevention. A quarter of the overall national burden are in the U5s. 
Across the six geopolitical zones, the highest burden was in children living in poorest rural households. Based on the variations in U5 malaria risk across regions in Nigeria, region-specific interventions will be more beneficial in reducing the burden of malaria than a one size fits all approach.

\section{Declarations}

\section{Competing interests}

None declared

\section{Authors' contributions}

VMO conceived the study, led data acquisition and analysis. VMO, AEA, AOA, OAE and ECM participated in interpretation of data for the work. AEA and AOA drafted introduction; VMO drafted methods/results; OAE and ECM drafted discussion. All authors participated in the revision of the entire manuscript for important intellectual content and approved the final manuscript for submission.

\section{References}

1. Cohee LM, Laufer MK. Malaria in Children. Pediatr Clin North Am. 2017;64(4):851-66.

2. Degarege A, Fennie K, Degarege D, Chennupati S, Madhivanan PJPo. Improving socioeconomic status may reduce the burden of malaria in sub Saharan Africa: A systematic review and metaanalysis. 2019;14(1).

3. WHO. Malaria Geneva, Switzerland: World Health Organization; 2020a [cited 2020. Available from: https://www.who.int/news-room/fact-sheets/detail/malaria.

4. Hershey CL, Florey LS, Ali D, Bennett A, Luhanga M, Mathanga DP, et al. Malaria Control Interventions Contributed to Declines in Malaria Parasitemia, Severe Anemia, and All-Cause Mortality in Children Less Than 5 Years of Age in Malawi, 2000-2010. Am J Trop Med Hyg. 2017;97(3_Suppl):76-88.

5. Smithson P, Florey L, Salgado SR, Hershey CL, Masanja H, Bhattarai A, et al. Impact of Malaria Control on Mortality and Anemia among Tanzanian Children Less than Five Years of Age, 1999-2010. PLoS One. 2015;10(11):e0141112.

6. Kayentao K, Florey LS, Mihigo J, Doumbia A, Diallo A, Koné D, et al. Impact evaluation of malaria control interventions on morbidity and all-cause child mortality in Mali, 2000-2012. Malar J. 2018;17(1):424.

7. WHO. World malaria report 2019. Geneva, Switzerland: World Health Organization. 2019.

8. Afoakwah C, Deng X, Onur I. Malaria infection among children under-five: the use of large-scale interventions in Ghana. BMC public health. 2018;18(1):536.

9. UNICEF. Malaria: United Nations Children's Fund; 2019 [cited 2020. Available from: https://data.unicef.org/topic/child-health/malaria/. 
10. Shretta R, Liu J, Cotter C, Cohen J, Dolenz C, Makomva K, et al. Malaria Elimination and Eradication. In: rd, Holmes KK, Bertozzi S, Bloom BR, Jha P, editors. Major Infectious Diseases. Washington (DC): The International Bank for Reconstruction and Development / The World Bank.; 2017.

11. National Malaria Elimination Programme, National Population Commission (NPopC), National Bureau of Statistics (NBS), ICF International. Nigeria Malaria Indicator Survey 2015. Abuja, Nigeria, and Rockville, Maryland, USA: NMEP, NPopC, and ICF International.; 2016.

12. Merlo J, Chaix B, Ohlsson H, Beckman A, Johnell K, Hjerpe P, et al. A brief conceptual tutorial of multilevel analysis in social epidemiology: using measures of clustering in multilevel logistic regression to investigate contextual phenomena. J Epidemiol Community Health. 2006;60(4):290-7.

13. Larsen K, Merlo J. Appropriate assessment of neighborhood effects on individual health: integrating random and fixed effects in multilevel logistic regression. American journal of epidemiology. 2005;161(1):81-8.

14. Konate D, Diawara SI, Toure M, Diakite SAS, Guindo A, Traore K, et al. Effect of routine seasonal malaria chemoprevention on malaria trends in children under 5 years in Dangassa, Mali. Malaria journal. 2020;19(1):137.

15. Chatio S, Ansah NA, Awuni DA, Oduro A, Ansah PO. Community acceptability of Seasonal Malaria Chemoprevention of morbidity and mortality in young children: A qualitative study in the Upper West Region of Ghana. PLoS One. 2019;14(5):e0216486.

16. Pitt C, Ndiaye M, Conteh L, Sy O, Hadj Ba E, Cissé B, et al. Large-scale delivery of seasonal malaria chemoprevention to children under 10 in Senegal: an economic analysis. Health Policy Plan. 2017;32(9):1256-66.

17. Cissé B, Ba EH, Sokhna C, JL ND, Gomis JF, Dial Y, et al. Effectiveness of Seasonal Malaria Chemoprevention in Children under Ten Years of Age in Senegal: A Stepped-Wedge ClusterRandomised Trial. PLoS Med. 2016;13(11):e1002175.

18. Nonvignon J, Aryeetey GC, Issah S, Ansah P, Malm KL, Ofosu W, et al. Cost-effectiveness of seasonal malaria chemoprevention in upper west region of Ghana. Malar J. 2016;15:367.

19. Abossie A, Yohanes T, Nedu A, Tafesse W, Damitie M. Prevalence of Malaria and Associated Risk Factors Among Febrile Children Under Five Years: A Cross-Sectional Study in Arba Minch Zuria District, South Ethiopia. Infect Drug Resist. 2020;13:363-72.

20. Samadoulougou S, Maheu-Giroux M, Kirakoya-Samadoulougou F, De Keukeleire M, Castro MC, Robert A. Multilevel and geo-statistical modeling of malaria risk in children of Burkina Faso. Parasit Vectors. 2014;7(1):350.

21. Roberts D, Matthews G. Risk factors of malaria in children under the age of five years old in Uganda. Malar J. 2016;15:246.

22. Spitzen J, Smallegange RC, Takken W. Effect of human odours and positioning of CO2 release point on trap catches of the malaria mosquito Anopheles gambiae sensu stricto in an olfactometer. Physiological Entomology. 2008;33(2):116-22. 
23. Boyle MH, Racine Y, Georgiades K, Snelling D, Hong S, Omariba W, et al. The influence of economic development level, household wealth and maternal education on child health in the developing world. Social science \& medicine. 2006;63(8):2242-54.

24. Degarege A, Fennie K, Degarege D, Chennupati S, Madhivanan P. Improving socioeconomic status may reduce the burden of malaria in sub Saharan Africa: A systematic review and meta-analysis. PLoS One. 2019;14(1):e0211205.

25. Yankson R, Anto EA, Chipeta MG. Geostatistical analysis and mapping of malaria risk in children under 5 using point-referenced prevalence data in Ghana. Malar J. 2019;18(1):67.

26. Ma C, Claude KM, Kibendelwa ZT, Brooks H, Zheng X, Hawkes M. Is maternal education a social vaccine for childhood malaria infection? A cross-sectional study from war-torn Democratic Republic of Congo. Pathog Glob Health. 2017;111(2):98-106.

27. Gone T, Lemango F, Eliso E, Yohannes S, Yohannes T. The association between malaria and malnutrition among under-five children in Shashogo District, Southern Ethiopia: a case-control study. Infect Dis Poverty. 2017;6(1):9.

28. Chitunhu S, Musenge E. Spatial and socio-economic effects on malaria morbidity in children under 5 years in Malawi in 2012. Spat Spatiotemporal Epidemiol. 2016;16:21-33.

29. Wilson AL, Bradley J, Kandeh B, Salami K, D'Alessandro U, Pinder M, et al. Is chronic malnutrition associated with an increase in malaria incidence? A cohort study in children aged under 5 years in rural Gambia. Parasit Vectors. 2018;11(1):451.

30. Temu EA, Coleman M, Abilio AP, Kleinschmidt I. High prevalence of malaria in Zambezia, Mozambique: the protective effect of IRS versus increased risks due to pig-keeping and house construction. PLoS One. 2012;7(2):e31409.

31. Maharaj R, Seocharan I, Qwabe B, Mkhabela M, Kissoon S, Lakan V. Decadal epidemiology of malaria in KwaZulu-Natal, a province in South Africa targeting elimination. Malar J. 2019;18(1):368.

32. Okullo AE, Matovu JKB, Ario AR, Opigo J, Wanzira H, Oguttu DW, et al. Malaria incidence among children less than 5 years during and after cessation of indoor residual spraying in Northern Uganda. Malar J. 2017;16(1):319.

33. Rumisha SF, Shayo EH, Mboera LEG. Spatio-temporal prevalence of malaria and anaemia in relation to agro-ecosystems in Mvomero district, Tanzania. Malar J. 2019;18(1):228.

34. Ferrão JL, Mendes JM, Painho M, João SZ. Spatio-temporal variation and socio-demographic characters of malaria in Chimoio municipality, Mozambique. Malar J. 2016;15:329.

35. Stebbins RC, Emch M, Meshnick SR. The Effectiveness of Community Bed Net Use on Malaria Parasitemia among Children Less Than 5 Years Old in Liberia. Am J Trop Med Hyg. 2018;98(3):6606.

36. Tusting LS, Bisanzio D, Alabaster G, Cameron E, Cibulskis R, Davies M, et al. Mapping changes in housing in sub-Saharan Africa from 2000 to 2015. Nature. 2019;568(7752):391-4.

37. Tusting LS, Bottomley C, Gibson H, Kleinschmidt I, Tatem AJ, Lindsay SW, et al. Housing Improvements and Malaria Risk in Sub-Saharan Africa: A Multi-Country Analysis of Survey Data. 
PLoS Med. 2017;14(2):e1002234.

Figures

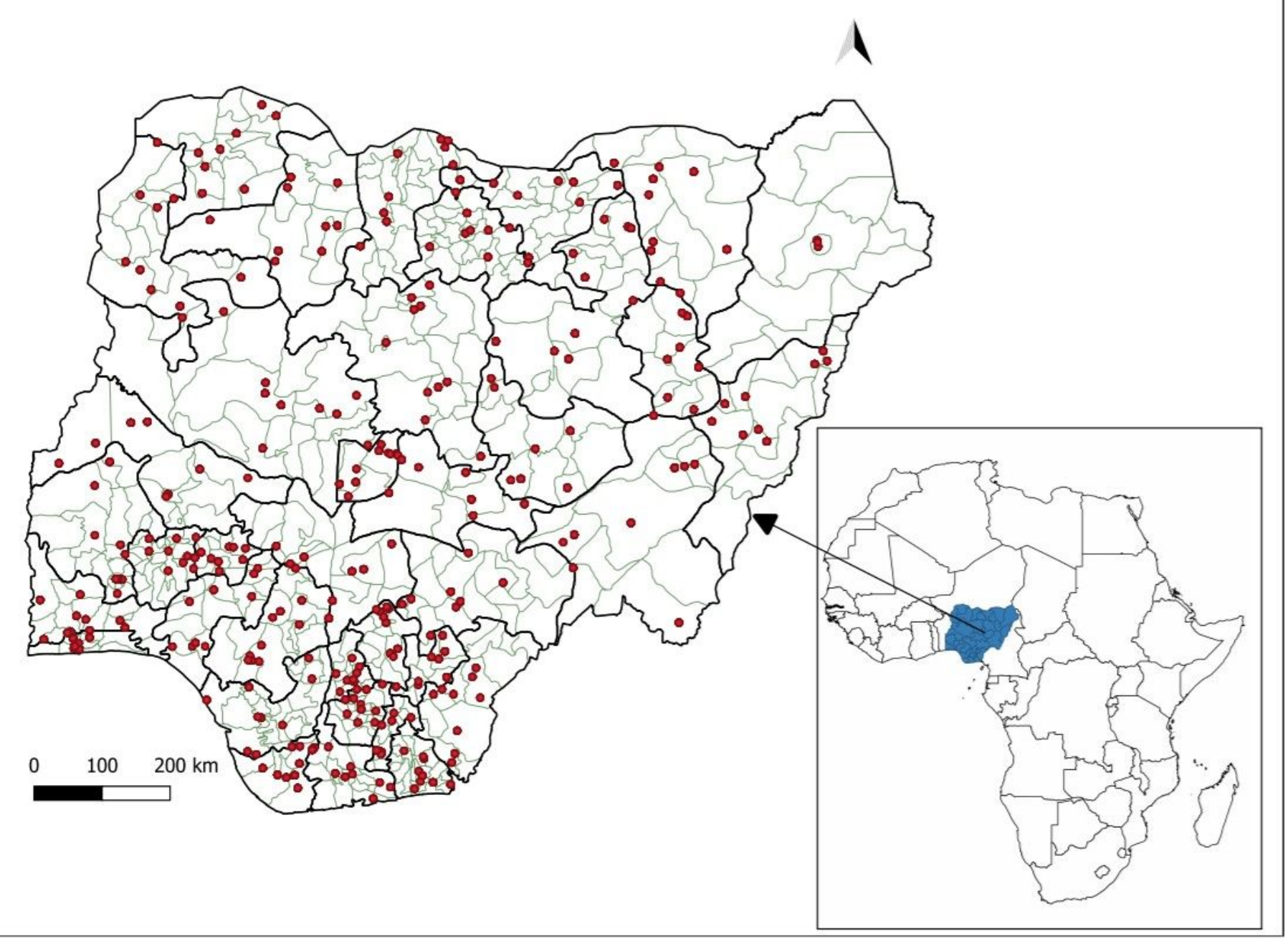

Figure 1

Map of Nigeria showing geographical location of the study, clusters and State boundaries 


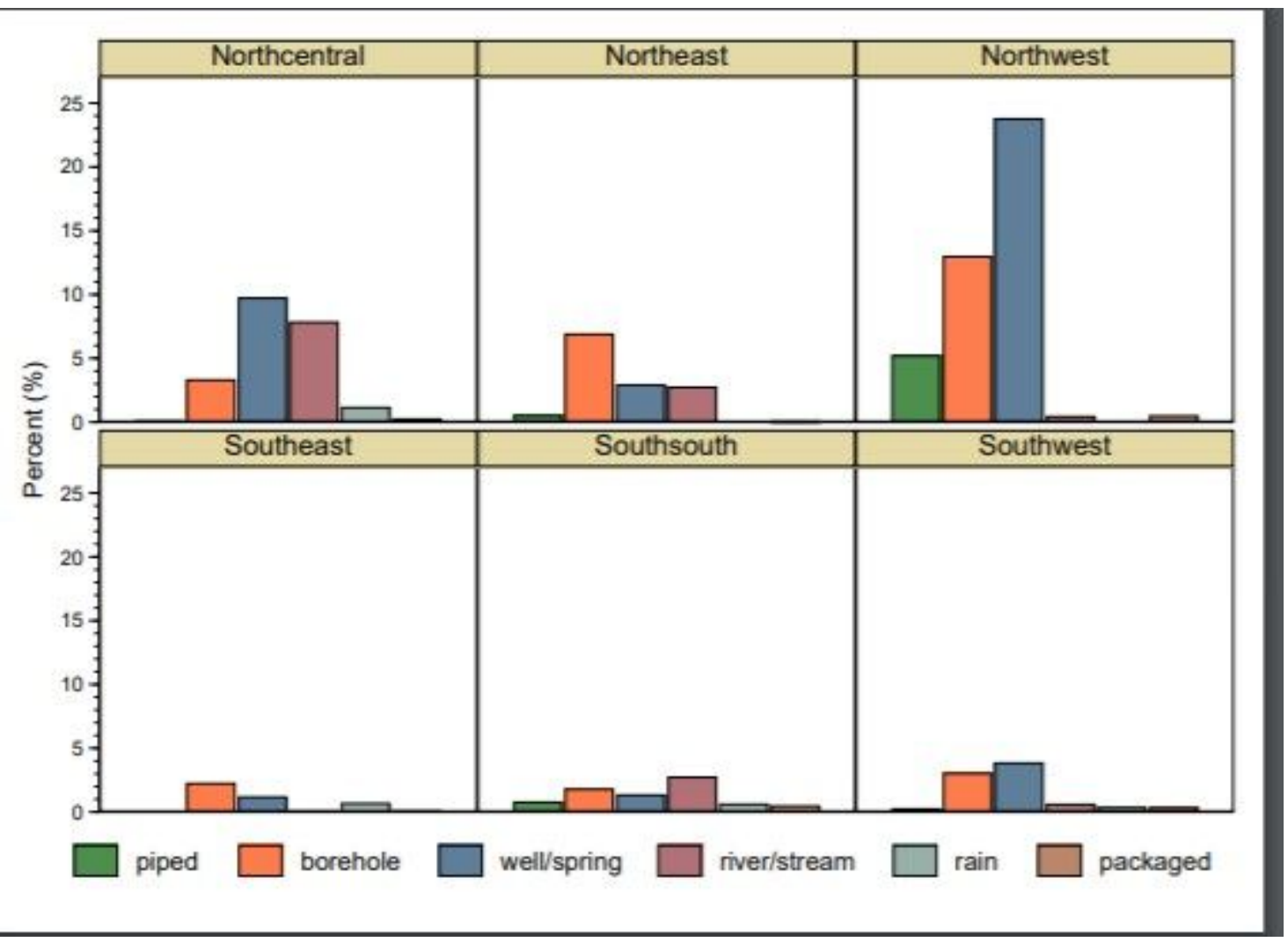

Figure 2

Prevalence of U5 Malaria by region and source of drinking water

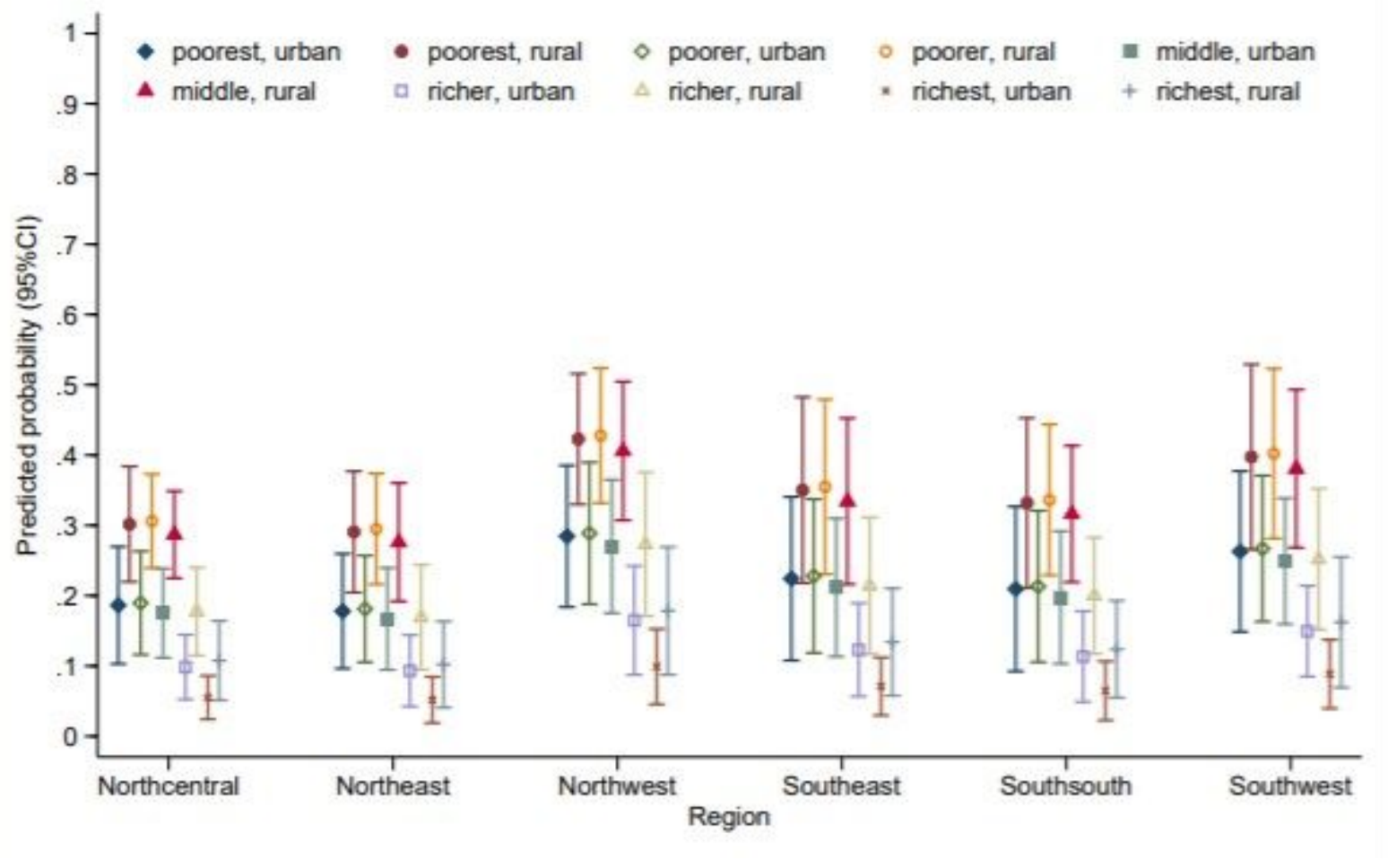

Figure 3 

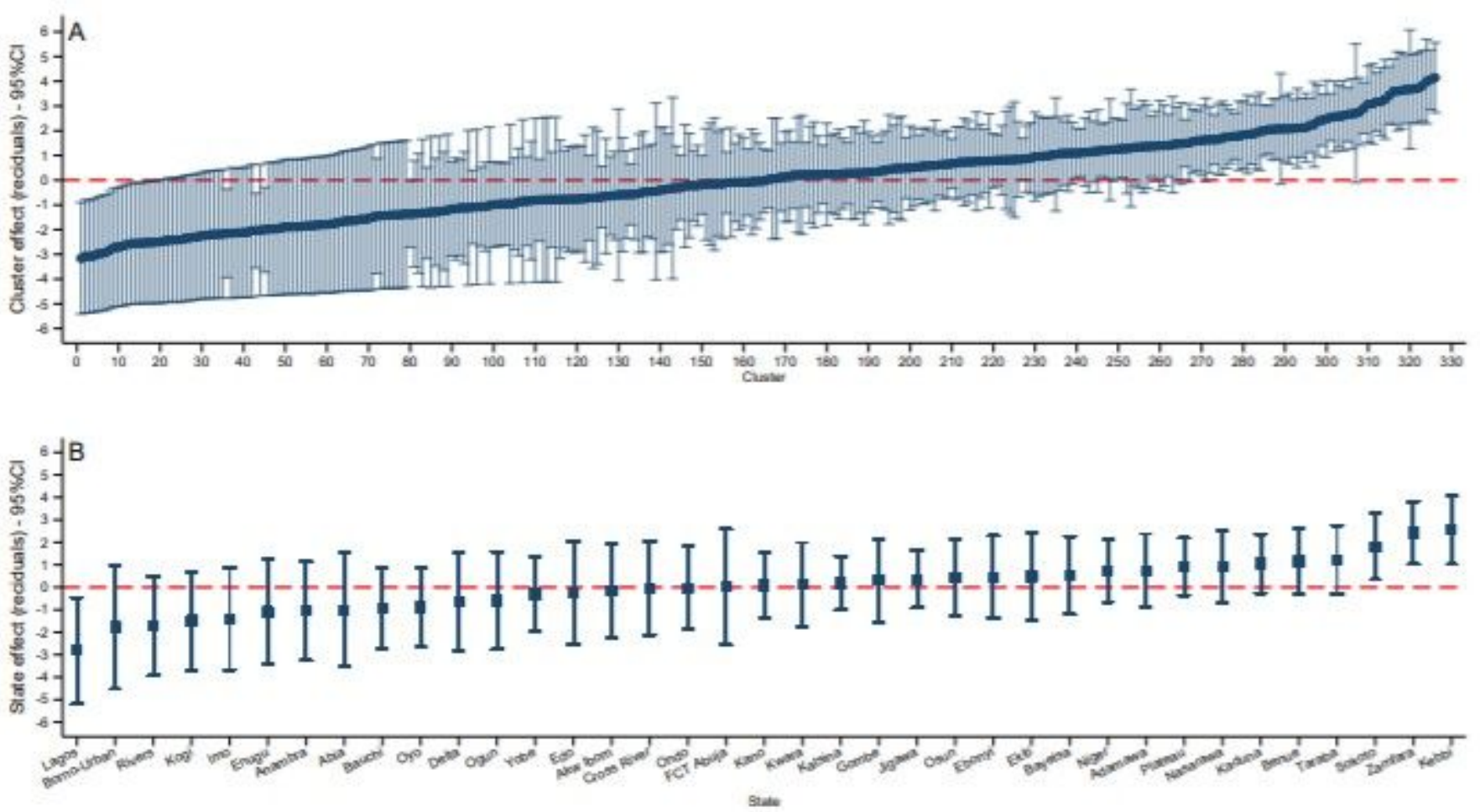

\section{Figure 4}

Cluster (A) and State (B) level variations in prevalence of malaria from the null model 